\title{
Jean-François H. Geschwind and Michael D. Dake (Eds): Abrams' Angiography: Interventional Radiology, 3rd edn
}

\author{
Lippincott Williams \& Wilkins, Philadelphia, 2014; ISBN 978-1-60913-792-2
}

\author{
Sara M. d. A. Giorgio • Luigi Mansi
}

Published online: 28 May 2014

(C) Springer-Verlag Berlin Heidelberg 2014

Angiography represents an ultraspecialized branch of radiology which is in constant evolution and requires skills and knowledge different and/or supplementary to those involved in "standard", i.e. not invasive, procedures. Because of the major risks and of possible negative results deriving from a mistake or a wrong therapeutic choice, all interventional radiologists have the duty to go beyond the basic principles of this discipline. For this purpose, the third edition of Abrams' Angiography: Interventional Radiology continues to represent a reference text, being one of the most complete and updated publications today available. Completely revamped to reflect the fast-moving landscape, the volume has been organized by two new editors, Jean-François H. Geschwind, professor of Radiology, Surgery, and Oncology at Johns Hopkins in Baltimore, and Michael D. Dake, professor of Cardiothoracic surgery at Stanford University. They recruited a team of 60 of the most renowned authorities in the field to focus on the 100 topics essential to mastering contemporary interventional radiology.

The book, consisting of 1,222 pages enriched by more than 1,100 full-colour illustrations, is divided into 16 sections and 100 chapters to offer a panoramic and comprehensive view on all fields of this independent radiological discipline.

The first section is dedicated to the interventional radiology in oncology and is divided into two parts. In part $A$ the therapy for organ-specific cancer is described, also considering epidemiology, staging, and medical and surgical management; part B addresses specialized interventional techniques in cancer care such as vascular access (venous or arterial ports), palliative care, and symptom management. The second section regards gynaecology and male fertility, describing issues such as uterine fibroids, uterine artery embolization,

S. M. d. A. Giorgio $\cdot$ L. Mansi $(\varangle)$

Second University of Naples, Caserta, Italy

e-mail: luigi.mansi@unina2.it pelvic congestion syndrome, postpartum pelvic haemorrhage, and the most common cause of male infertility, i.e. varicocele. The third section deals with vascular anomalies, analyzing hemangiomas and vascular malformations. The fourth section describes how interventional radiology may approach liver diseases, describing trans-jugular intra-hepatic porto-systemic shunts, trans-jugular liver biopsy and retrograde balloon occlusion variceal ablation. The remaining sections of the text (from V to XVI) are dedicated to the management of arterial and venous diseases such as arterial and venous occlusive diseases, vasculitides, arteriopathies, aneurysms, haemorrhage, embolic and thrombo-embolic obstruction, traumatic injuries, transplants, and also includes less frequent diseases, noninvasive diagnostic evaluations, and more specialized indications. Finally, in the latest section, the authors examine pharmacologic therapies in depth, i.e. the interaction between the foreign materials being implanted and the human body, in particular considering medical therapies.

This text, a milestone of angiography, has now been expanded and thoroughly revised to reflect dynamic advances in this discipline using a clear writing style understandable both by beginners and more expert radiology interventionists. The hundreds of high quality images, many of which are in brilliant colours, are combined with complete and easily understandable captions. Each chapter is accompanied by a valuable bibliography, which allows those who wish to deepen their knowledge with the appropriate documentation subject matter.

In conclusion, Abrams' Angiography: Interventional Radiology still represents a reference that will be useful to any specialist who performs or is interested in starting to perform interventional radiology. The presence of this book could also certainly be useful in the library of a department of nuclear medicine. 